\title{
Dissecting the Cellular Behaviour of Colorectal Cancer via Multimodal Imaging and Correlative Microscopy
}

\author{
Filip Braet ${ }^{1}$
}

\author{
1. Australian Centre for Microscopy \& Microanalysis and School of Medical Sciences, Discipline of \\ Anatomy \& Histology, The University of Sydney, Sydney, Australia
}

These days, the common ground between structural cell biology and molecular biology continues to grow thanks to the biomolecular insights offered by correlative microscopy (CM). CM utilizes the latest advances in contemporary multimodal imaging approaches that are now capable of filling in the missing pieces of the puzzle. CM allows us to interrogate a sample's fine structure from the nano- to micrometre scale in a way that is impossible with any one technique alone [1]. The rapidly expanding CM toolset delivers a strong degree of confidence in the observed structures of interest as insights obtained from one microscopy approach can be directly interrogated by other imaging modalities [2].

Here, a versatile multimodal CM workflow encompassing vastly different temporal and spatial resolutions will be presented to reveal novel structural-functional information in colorectal cancer (CRC)-mediated events within hepatic tissue, including liver sinusoidal cells. Specifically, we will correlate molecular- and cellular dynamics data, along with inter- and intra-cellular signalling events of hepatic colorectal metastases obtained by multimodal CM using cross-integrative analysis. The multimodal imaging approaches utilised in this study include: live animal and live cell imaging [3], microcomputed tomography [4], wide-field and super-resolution nanoscopy [5], correlative fluorescence scanning and transmission electron microscopy [6,7], atomic force probing, and 3-D large- and smallvolume electron tomography [8,9] (Figure 1). Furthermore, we will discuss the careful selection of biomolecular labels for cross-correlative imaging within the same sample of the model organism of interest.

These multimodal imaging and CM approaches added the following insights: (i) metastasizing CRC cells are preferentially restrained in the periportal sinusoids of liver tissue due to their size; (ii) macrophages, natural killer cells, and endothelial cells work in concert as one immune-surveillance guardian in the removal of the majority of the CRC cells, thereby controlling tumour growth; (iii) 5\% of the CRC cells are able to escape the local hepatic immune system and provide themselves a gateway towards the liver parenchymal tissue by inducing apoptosis and retraction of the endothelium; (iv) the apoptosis regulating molecules Fas/FasL play a key role in the metastatic cascade; (v) the IFN $\gamma / \mathrm{NO}$ pathway has been proven to be a promising molecular target for the development of a new immunotherapeutic strategy in CRC; and, (vi) surviving cancer cells are primarily confined to the Space of Disse and to the Glisson capsule, suggesting that further tumour proliferation and growth are likely to initiate from these extracellular matrix-rich regions of the liver.

By drawing on these novel correlative image models, we are finally beginning to understand the mechanisms of CRC metastasis that will enable us to strategically design alternative therapeutic approaches for the treatment of colorectal liver metastases and assess their efficacy [10]. Key approaches presently under investigation involve various organic and inorganic complexes, including macrocomplexes innate to the body, which show selective activity towards colorectal cancer cells [11]. Those supramolecular complexes can respectively be used as a delivery vehicle for current anti-cancer 
drugs or as theranostic compounds.

References:

[1] Y Su et al, Biophys Rev 2 (2010) 121.

[2] KA Jahn et al, Micron 43 (2012) 565.

[3] K Kobayashi et al, Methods Cell Biol 111 (2012) 1.

[4] D Cheng et al, Biomed Res Int (2016) 8758460.

[5] F Braet et al, Methods Cell Biol 124 (2014) 129.

[6] CPH Vreuls et al, Hepatology 59 (2014) 737.

[7] G Shami et al, Sci Rep 6 (2016) 36744.

[8] G Shami et al, J Cell Physiol 232 (2017) 249.

[9] D Cheng et al, Methods Cell Biol In press (2017) 34.

[10] KA Jahn et al, J Pharm Sci 100 (2011) 3751.

[11] YN Wu et al, Int J Nanomed 8 (2013) 3321.

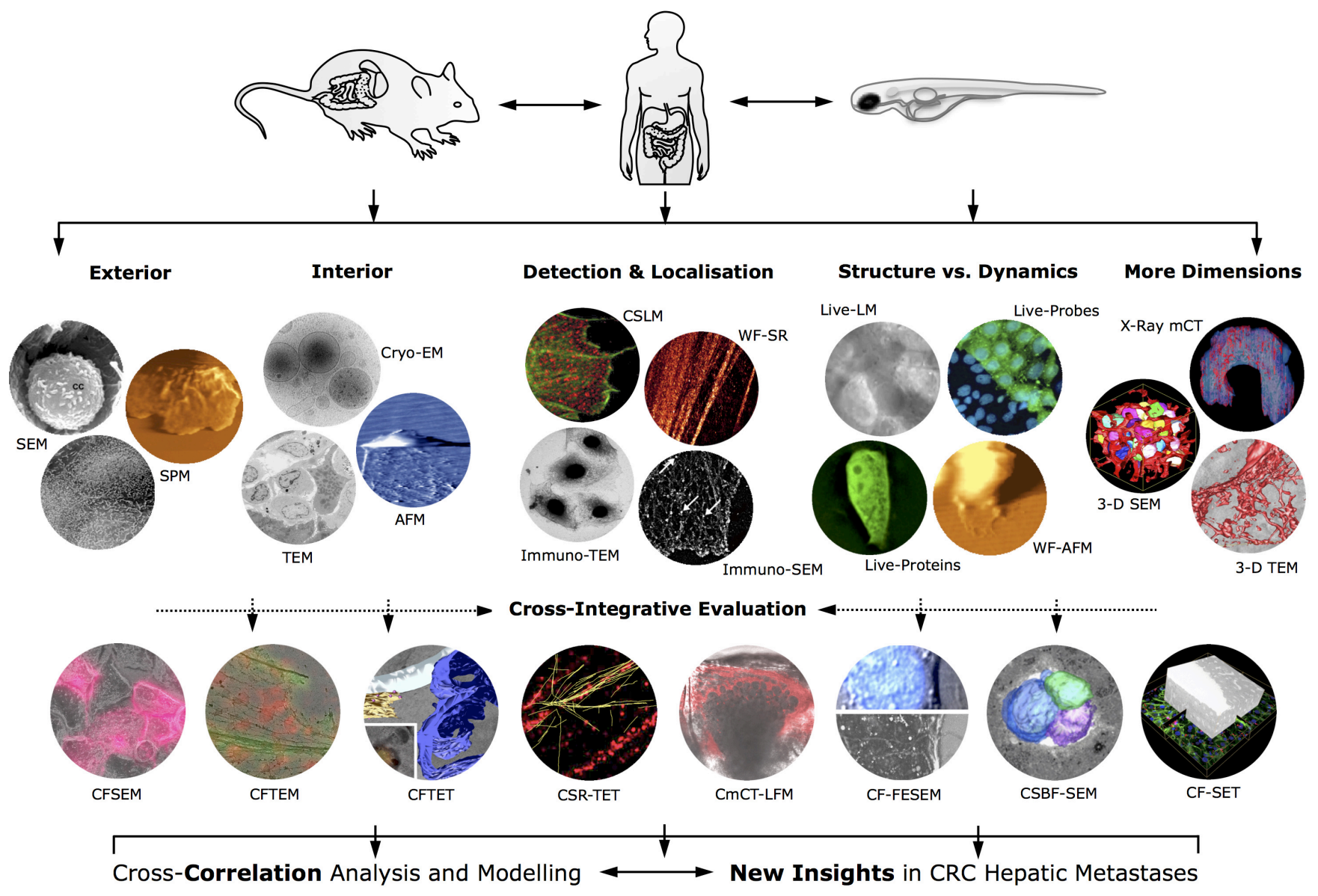

Figure 1. Schematic overview depicting the multimodal imaging workflow employed to disclose colorectal cancer cell behaviour in various experimental models such as rodents, zebrafish and human tissue. Note the use of different beam-lines-i.e., X-rays, photons, force and electrons - permitting to accrue correlative morphology-function evidence by employing a diverse rang of biomolecular labeling and imaging methodologies to the same sample, also known as 'correlative morphometrics' 